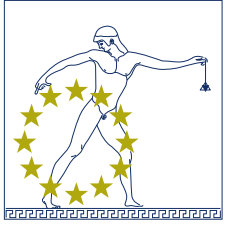

EURAPS

\title{
Health-related quality of life after oncological resection and reconstruction of the chest wall
}

\author{
J.T.K. Salo ${ }^{\mathrm{a}, *}$, J.P. Repo ${ }^{\mathrm{a}, \mathrm{b}}$, R.P. Roine ${ }^{\mathrm{c}, \mathrm{d}}$, H. Sintonen ${ }^{\mathrm{e}}$, \\ E.J. Tukiainen ${ }^{\text {a }}$
}

a Department of Plastic Surgery, University of Helsinki and Helsinki University Hospital, Helsinki, Finland

${ }^{b}$ Department of Surgery, Central Finland Health Care District, Jyväskylä, Finland

'Group Administration, University of Helsinki and Helsinki University Hospital, Finland

${ }^{\mathrm{D}}$ Department of Health and Social Management, Research Centre for Comparative Effectiveness and

Patient Safety, University of Eastern Finland, Kuopio, Finland

' Department of Public Health, University of Helsinki, Helsinki, Finland

Received 17 December 2018; accepted 16 May 2019

Available online $x x x$

\section{KEYWORDS \\ Quality of life; \\ Chest wall; \\ Reconstruction; \\ Resection; \\ 15D; \\ QLQ-C30; \\ Sarcoma; \\ Breast cancer}

\begin{abstract}
Summary Objectives: There is limited information of the health-related quality of life (HRQoL) after surgical treatment of chest wall tumors. This cross-sectional study aimed to assess long-term HRQoL after chest wall reconstruction following oncological resection.

Methods: Seventy-eight patients having undergone chest wall tumor resection and reconstruction during 1997-2015 were invited to complete the 15D and QLQ-C30 HRQoL instruments.

Results: Altogether, 55 patients (17 men and 38 women), with a mean (SD) age of 68 (14) years, completed the questionnaires (response rate $71 \%$ ). Patients had been operated due to soft tissue sarcoma $(n=16)$, advanced breast cancer $(n=15)$, osteo- or chondrosarcoma $(n=14)$, or other tumor $(n=10)$. Median time after primary surgery was 66 (IQR 38,141$)$ months. The resection was full thickness in 29/55 cases and partial thickness in $26 / 55$ cases. Chest wall reconstruction was required for $47 / 55$ cases (85\%). Reconstruction was performed using softtissue flap in eight cases, skeletal stabilizations with mesh or mesh-cement-mesh (sandwich method) in 15 cases, and skeletal stabilizations and soft-tissue flap in 24 cases.

Patients' mean 15D score $(0.878$, SD 0.111$)$ was comparable to that of the age- and genderstandardized general population (0.891, SD 0.041). Limitations in breathing and usual activities were noted. The QLQ-C30 cancer-specific HRQoL was 72 points (maximum 100). Scores in the QLQ-C30 Functional scales ranged from 78 (Physical) to 91 (Social).
\end{abstract}

\footnotetext{
* Correspondence to: Department of Plastic Surgery, Töölö Hospital, PO Box 266, FIN 0029 HUS, Finland.

E-mail address: juho.salo@hus.fi (J.T.K. Salo).
} 
Conclusions: Long-term HRQoL in patients after chest wall reconstruction following oncological resection is fair and comparable to that of the general population. Limitations in breathing and usual activities can occur.

(C) 2019 British Association of Plastic, Reconstructive and Aesthetic Surgeons. Published by Elsevier Ltd. All rights reserved.

\section{Introduction}

Chest wall resection and reconstruction are a surgical challenge. Large chest wall oncological tumor resection and reconstruction are indicated because of a primary, locally invading, or metastatic tumor. The most common oncological indications for chest wall resection are bone and cartilage tumors, soft tissue sarcomas, and advanced lung and breast cancer. ${ }^{1,2}$

Surgical operations include full-thickness or partialthickness resection and immediate reconstruction of the chest. The method of reconstruction depends on the anatomical site, depth, and size of the defect. Reconstruction is usually performed with chest wall stabilization, soft tissue flap coverage, or a combination of both. ${ }^{3}$ Some patients also receive oncological adjuvant treatment. ${ }^{4}$

With modern operative techniques, coupled with perioperative and postoperative treatments, even extensive chest wall operation can be safely performed with favorable results. Severe postoperative morbidity is uncommon, and the oncological results are acceptable. The long-term survival rates of the patients depend on the varying biological behaviors of the underlying malignancies; chondro/osteosarcoma, soft tissue sarcoma, advanced breast, or lung cancer. ${ }^{5}$ Furthermore, the presence of metastases, the surgical results, adjuvant oncological treatments, patient material, and selection all have an effect on long-term survival. ${ }^{5,6}$

There is limited information of the long-term, healthrelated quality of life (HRQoL) after surgical treatment of chest wall-related tumors with chest wall resection and reconstruction. Assessing HRQoL can provide important information about the treatment outcomes of chest wall tumors. As a patient-reported outcome, the HRQoL status can be used to express patients' opinions concerning their physical, mental, emotional, and social well-being. Recently, HRQoL research has been increasingly integrated into cancer studies, and HRQOL has become an important end point of care. $^{7}$

Few previous studies assessing HRQoL in patients after chest wall tumor resection all have some limitations. ${ }^{8-12}$ They are hampered by small sample size $\mathrm{e}^{8,9,11,12}$; fail to compare results obtained in patients to those of a healthy control population ${ }^{8,10-12}$; or do not report the extent of the surgical operations, need for reconstruction, and methods of reconstruction. ${ }^{9,10}$ There are no studies assessing postoperative HRQoL of patients with chest wall sarcoma. Additionally, information of HRQoL after surgery is limited in chest wall-related advanced breast-cancer. ${ }^{13}$

The aim of this study was to assess, using the 15D and the QLQ-C30 HRQoL instruments, the long-term HRQoL in patients after chest wall reconstruction following oncological resection.

\section{Patients and methods}

\section{Patients}

The hospital electronic database at the Department of Plastic Surgery, Helsinki, Finland, was searched for adult patients who had undergone oncological chest wall resection and reconstruction between January 1, 1997, and December 31, 2015. Exclusion criteria were infected sternotomy or other chest wall infection, simple benign tumor excision with direct closure, congenital chest wall deformity, and bronchopleural fistula. The minimum follow-up time was one year. Altogether, 135 patients met the inclusion criteria. The demographic data for these 135 patients have been described elsewhere in more detail. ${ }^{5}$

By the start of HRQol data collection in February 2016, 55 (41\%) of the 135 surgical patients had died and two patients had moved overseas. Two HRQoL questionnaires together with a questionnaire consisting of items about sociodemographic and clinical details were mailed to the remaining 78 patients cross-sectionally. The patients were asked to sign an informed consent if they were willing to participate in the study and to return the questionnaires by mail in a prepaid envelope. If the patient failed to reply within a 3-week period, a reminder letter, together with a new set of questionnaires, was sent; 55 patients (71\%) answered the questionnaires.

Postoperative complications of the patients were classified according to the Clavien-Dindo classification. ${ }^{14}$ The classification consists of seven complication grades (I, II, IIIa, IIIb, IVa, IVb, and V). The grades are based on the severity and the type of therapy needed to correct the complication.

HRQoL results for the patients were compared with those of an age- and gender-standardized sample of the general population ( $n=1307$ ) obtained from the Health 2011 Survey in the hospital catchment area. ${ }^{15}$

\section{Instruments}

\section{The 15D questionnaire}

Generic HRQoL was measured using the 15D, ${ }^{16}$ a comprehensive instrument that covers 15 dimensions: mobility, vision, hearing, breathing, sleeping, eating, speech, excretion, usual activities, mental function, discomfort/symptoms, depression, distress, vitality, and sexual activity. Respondents rate each dimension on a scale from one (no problems) to five (severe problems).

The 15D produces both an HRQoL profile, based on dimension level values, and a single index score, which represents the overall HRQoL. Both are generated by incorporating population-based preference weights into 
the dimensions. The dimension-level values and the single index score are on a zero to one scale, where zero stands for being dead and one for best possible HRQoL (15D score) or no problems on the dimension (dimension-level value). Estimates place the test-retest reliability and minimal clinically important difference of the $15 \mathrm{D}$ score at 0.90 and 0.015 , respectively. ${ }^{17,18}$ The $15 \mathrm{D}$ is comparable favorably to other similar, generic HRQoL instruments in their most important psychometric properties. ${ }^{16,17,19-21}$

\section{EORTC-QLQ-C30 questionnaire}

The European Organisation for Research and Treatment of Cancer (EORTC) QLQ-C30 questionnaire is a standardized and self-administered HRQoL instrument designed for use in the estimation of the HRQoL in oncologic patients. The QLQ-C30 incorporates nine multi-item scales, including five functional scales, three symptom scales, a global health and quality of life scale, and six single-item symptom measures. A scale of items is scored from zero to 100 ; in the functional, the global healthy, and quality-oflife scales, higher scores indicate better health. In the symptom scales, higher score indicates more symptoms. ${ }^{22}$ Single-symptom items are scaled in the following manner: no symptoms = zero; mild symptoms $\leq 33.33$; moderate symptoms $\leq 66.66$; and severe symptoms $\leq 100$. Multi-item symptoms are scaled in the following manner: zero $=$ no symptoms; 0.01-66.65 = mild symptoms; 66.66$99.99=$ moderate symptoms; and $100=$ severe symptoms.

\section{Sociodemographic and clinical questionnaire}

Information was obtained about participants' age and sex, weight, length, comorbidities, medication, smoking habits, family circumstances, and occupational status.

\section{Statistical analysis}

The characteristics of the study population are presented as mean values with standard deviations (SD), as medians with interquartile range (IQR), or as counts with percentages. Hot-deck imputation was performed for missing data when needed. Statistical comparison between groups was performed by Fisher-Freeman-Halton test, $t$-test, permutation test, or bootstrap-type analysis of variance (ANOVA), when appropriate. Adjusted comparison between groups was evaluated using bootstrap-type analysis of covariance (ANCOVA). Models included sex, age, and Charlson comorbidity index as covariates. The bootstrap method was used when the theoretical distribution of the test statistics was unknown or in the case of violation of the assumptions (e.g., non-normality). Adjusted correlation (partial) coefficients were calculated by the Pearson method. Stata 15.1, StataCorp LP (College Station, TX, USA) statistical package was used for the analyses.

\section{Ethical considerations and reporting}

The study protocol was reviewed and approved by the Ethics Committee of the Hospital District of Helsinki and Uusi-
Table 1 Patients' sociodemographic and clinical characteristics $(n=55)$.

\begin{tabular}{ll}
\hline Mean age, years, (SD) & $68.3(14.4)$ \\
Female, $n$ (\%) & $38(69 \%)$ \\
Male, $n(\%)$ & $17(31 \%)$ \\
Body Mass Index, kg/m², (SD) & $26.0(4.2)$ \\
Median time after primary surgery, & $66(16-241,67)$ \\
$\quad$ months (range, SD) & \\
Smokers & \\
Yes, $n(\%)$ & $6(11 \%)$ \\
No, $n$ (\%) & $49(89 \%)$ \\
Comorbidities & \\
Hypertension, $n$ (\%) & $24(44 \%)$ \\
Diabetes, $n(\%)$ & $10(18 \%)$ \\
Coronary heart disease, $n(\%)$ & $5(9 \%)$ \\
Asthma, $n(\%)$ & $5(9 \%)$ \\
Thyroid disease, $n$ (\%) & $5(9 \%)$ \\
Other, $n(\%)$ & $6(11 \%)$ \\
Family circumstances & \\
Married/cohabitation, $n(\%)$ & $29(53 \%)$ \\
Widow, $n(\%)$ & $10(18 \%)$ \\
Divorced, $n(\%)$ & $7(13 \%)$ \\
Single, $n(\%)$ & $5(9 \%)$ \\
Occupational status & \\
Retired, $n(\%)$ & $41(75 \%)$ \\
Employment status, $n(\%)$ & $13(24 \%)$ \\
Unknown, $n(\%)$ & $1(1 \%)$ \\
\hline
\end{tabular}

maa, Helsinki, Finland. A written informed consent was obtained from all patients. Results are reported adhering to the Strengthening the Reporting of Observational Studies in Epidemiology (STROBE) statement. ${ }^{23}$

\section{Results}

\section{Chest wall resection and reconstruction patients}

A total of 17 men and 38 women (response rate $71 \%$ ) with a mean (SD) age of 68 (14) years completed the questionnaires. The median (range, SD) time after primary surgery was 66 (16-241, 67) months. Table 1 lists the sociodemographic and clinical characteristics of these patients.

The respondents had been operated because of soft tissue sarcoma $(n=16)$, advanced breast cancer $(n=15)$, osteo- or chondrosarcoma $(n=14)$, or another tumor $(n=10)$ of the chest wall. Resections of the chest wall were full thickness in 29 cases and partial thickness in 26 cases. All patients had undergone one-stage surgery including tumor removal and defect reconstruction in the same procedure. In eight patients, the defect could be closed primarily, whereas the remaining 47 (85\%) patients required chest wall reconstruction: soft tissue flap reconstruction in eight cases (Figure 1(a)-(c)), skeletal stabilization with mesh or meshcement-mesh in 15 cases (sandwich method), and skeletal stabilization and soft tissue flap reconstruction in 24 cases (Figure 2(a)-(d)). Oncological adjuvant treatment was given to $23 / 55(55 \%)$ of the patients. 

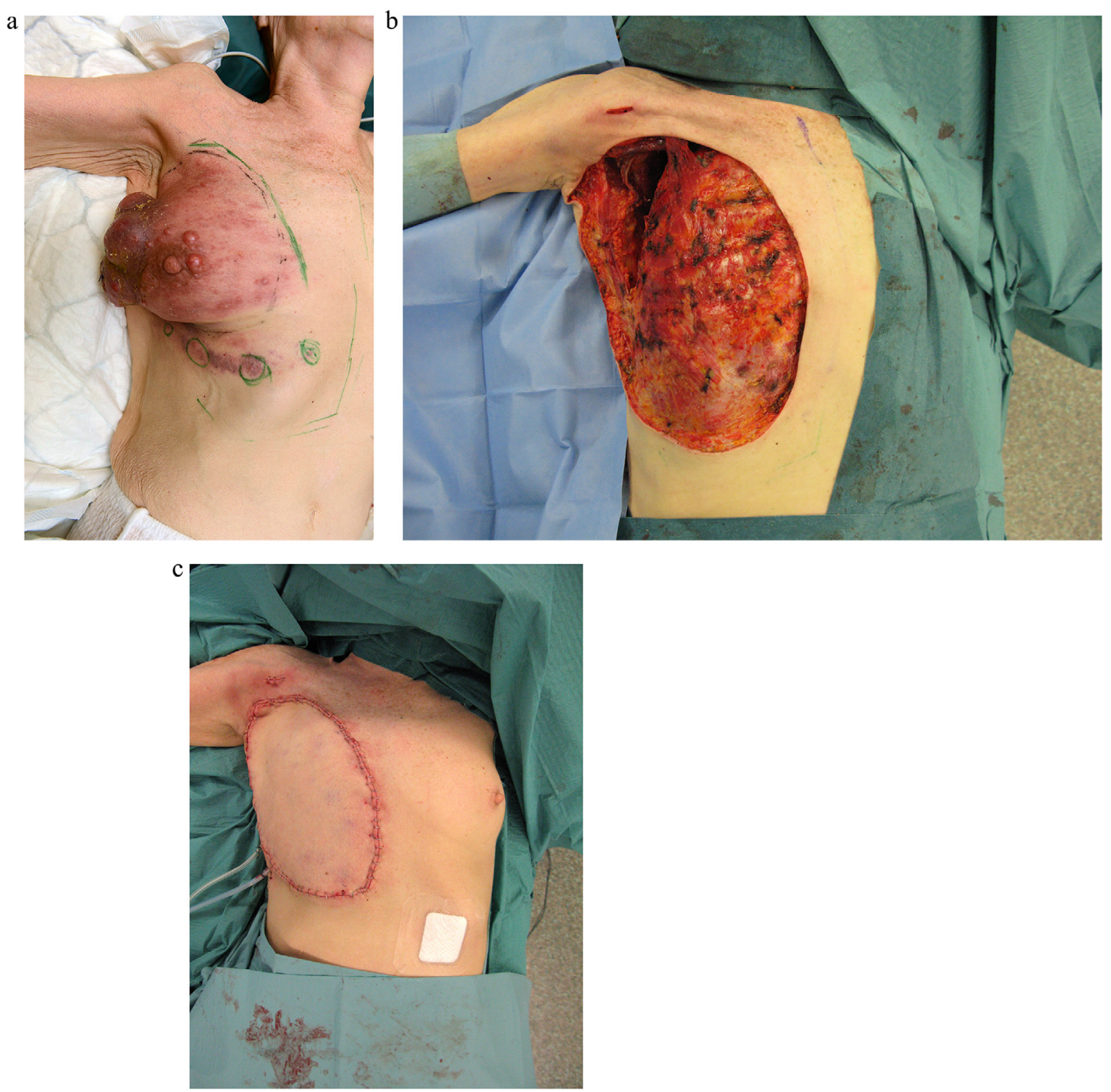

Figure 1 (a) Advanced breast cancer. (b) Partial-thickness anterolateral chest wall resection. (c) Chest wall reconstruction with free musculocutaneus tensor fascia flap.

In the majority of patients $(n=46,84 \%)$, no surgical complications were observed. Complications occurred in nine cases and three re-operations were needed. Complications were classified with the Clavien-Dindo classification: grade II $(n=5)$; grade IIIb $(n=2)$, or grade IVa $(n=2)$. The most common complication was pneumonia $(n=3)$. During follow-up, four patients developed recurrence of disease and four developed metastases.

\section{Comparison of respondents and nonrespondents}

On comparison of respondents $(n=55)$ and nonrespondents $(n=23)$, there were statistically significant differences in mean age; the respondents were seven years older on average. There were no statistically significant differences between the two groups for other characteristics.

\section{Comparison of different reconstruction type groups}

Table 2 shows the comparison of subject and operative characteristics in different reconstruction type groups. There was a statistically significant difference in the Charlson comorbidity index, diagnosis, and full-thickness resection.

\section{HRQoL (15D) of patients compared to that of control population}

The patients' mean $15 \mathrm{D}$ score $(0.878$, SD 0.111$)$ was comparable to that of the age- and gender-standardized general 

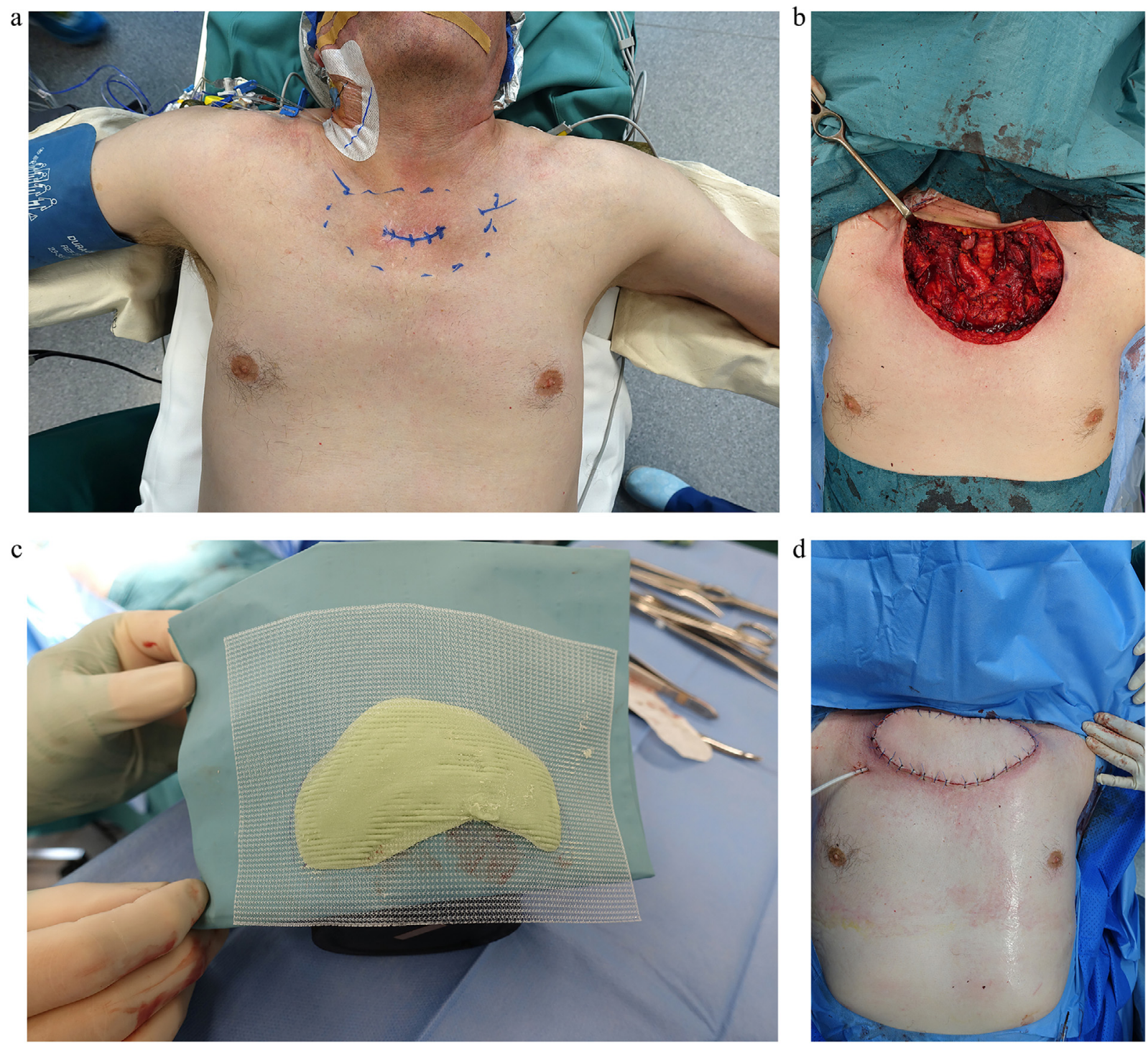

Figure 2 (a) Chest wall high grade soft tissue sarcoma. (b) Full-thickness anterior chest wall resection. (c) Chest wall stabilization with a sandwich technique (methyl-methacrylate between two meshes). (d) Soft tissue reconstruction with pedicled latissimus dorsi musculocutaneous flap.

population $(0.891$, SD 0.041). The difference was neither statistically significant nor clinically important. However, patients were statistically significantly worse off on the dimensions of "Breathing" $(p=0.043)$ and "Usual activities" $(p=0.027)$ (Figure 3$)$.

\section{QLQ-C30 scores}

Among all patients, the QLQ-C30 Global health status (HRQoL) was 72 points (zero to 100 , worst to best). Scores in the QLQ-C30 functional scales ranged from 78 (Physical) to 91 (Social) points (Figure 4).

Scores in the Symptom scales (zero to 100, best to worst) ranged from 2 (Nausea/vomiting) to 23 (Fatigue) points (Figure 5).

\section{Comparing quality of life (15D and QLQ-C30) in different reconstruction type groups}

In nonadjusted analyses, the mean 15D scores differed in a statistically significant manner on the dimensions of "Seeing" $(p=0.003)$, "Speech" $(p=0.014)$, and "Distress" $(p=0.024)$. When analyses were adjusted for sex, age, and Charlson comorbidity index, there were no statistically significant differences. There were no statistically significant differences in the mean EORTC QLQ-C30 symptom scores between the different reconstruction type groups (Table 3 ).

\section{Comparing quality of life and mean defect size}

There was a partial correlation with the 15D and the QLQC30 and with mean defect size when the analyses were adjusted for age, sex, and Charlson comorbidity index. 


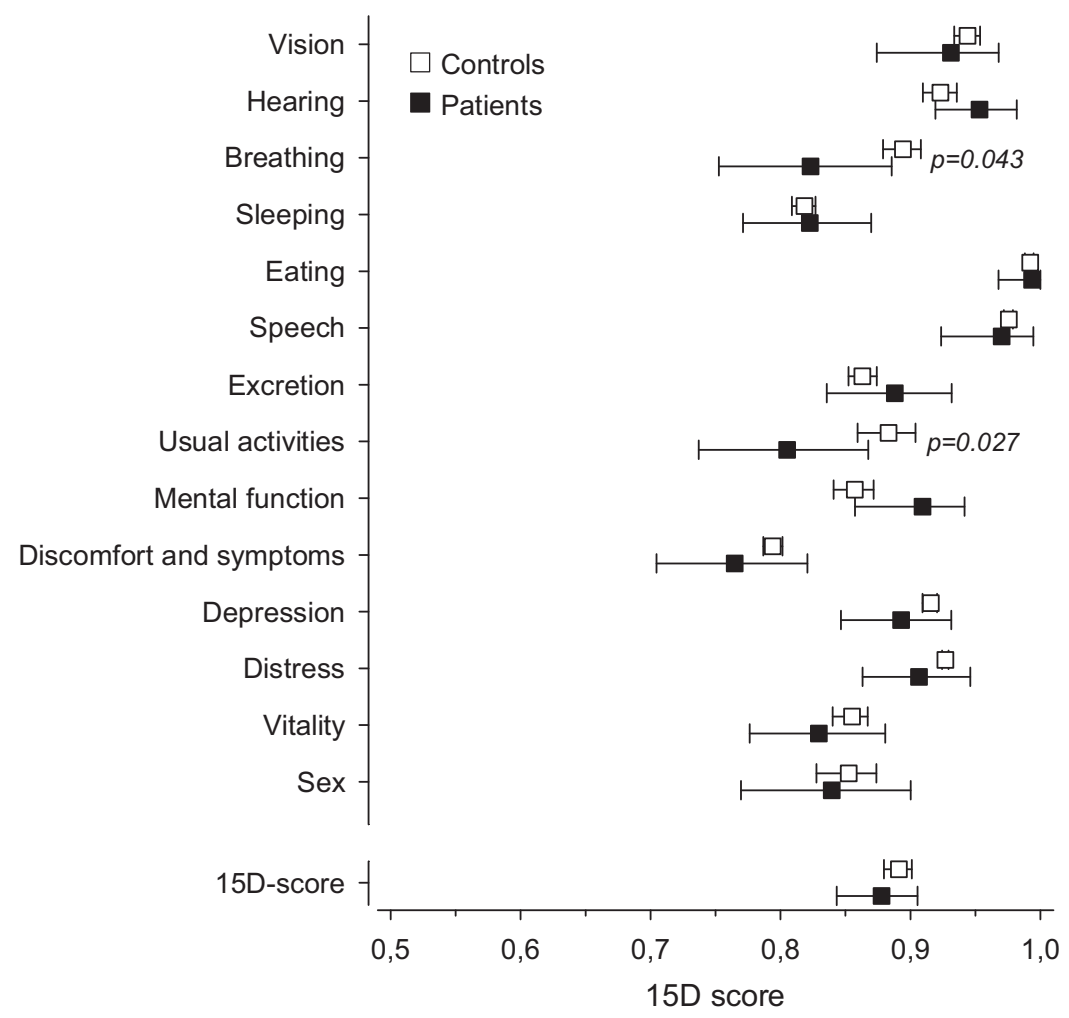

Figure 3 The mean 15D dimension scores and the total 15D score of patients who underwent chest wall reconstruction $(n=55)$ compared to those of the age- and gender-standardized general population.

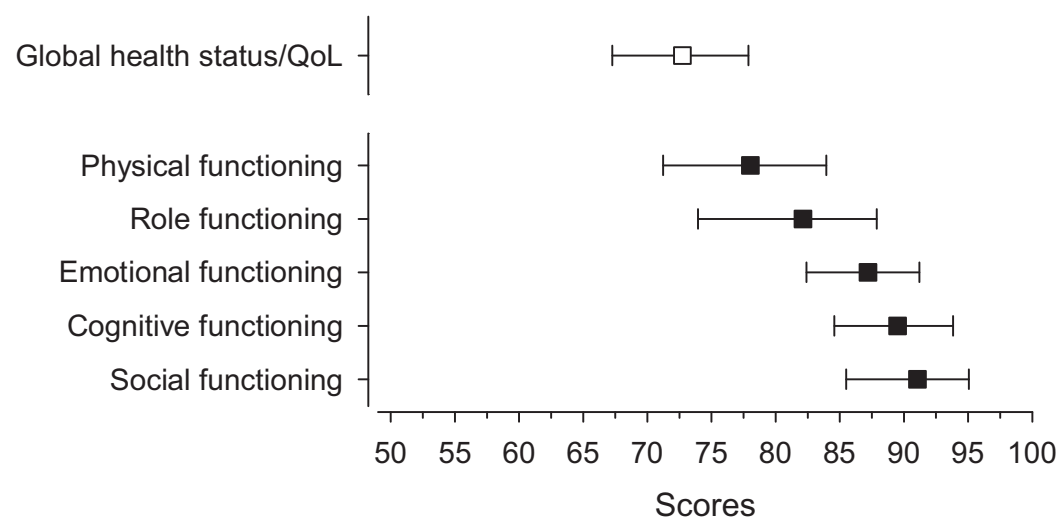

Figure 4 Mean QLQ-C30 function scores in patients who underwent chest wall reconstruction.

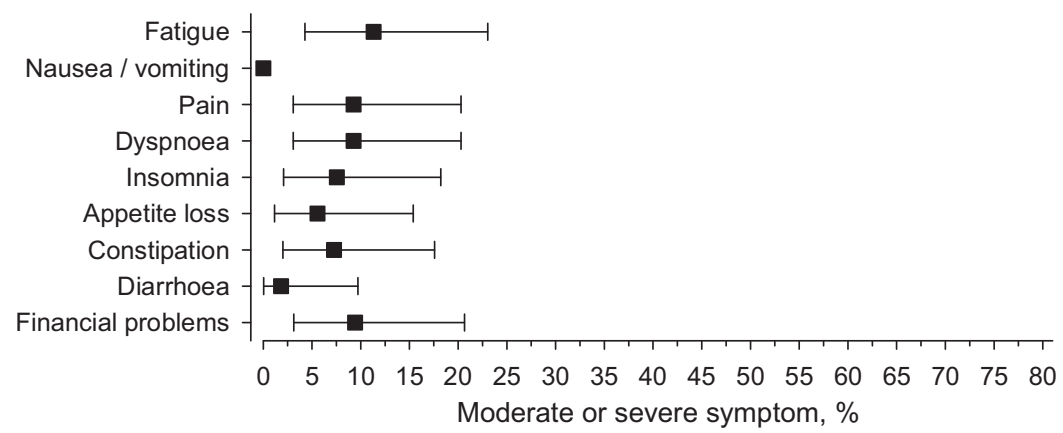

Figure 5 Prevalence of moderate or severe symptoms in patients who underwent chest wall reconstruction. 
Table 2 Subject and operative characteristics in different reconstruction type groups.

\begin{tabular}{|c|c|c|c|c|c|}
\hline & \multicolumn{4}{|c|}{ Reconstruction type } & \multirow[t]{2}{*}{$p$-value } \\
\hline & $\begin{array}{l}\text { Primary } \\
\text { closure } \\
n=8\end{array}$ & $\begin{array}{l}\text { Chest wall } \\
\text { stabilization } \\
n=15\end{array}$ & $\begin{array}{l}\text { Soft tissue } \\
\text { flap cover } \\
n=8\end{array}$ & $\begin{array}{l}\text { Chest wall } \\
\text { stabilization + flap } \\
n=24\end{array}$ & \\
\hline Mean age, years, (SD) & $72(8)$ & $62(17)$ & $75(13)$ & $67(14)$ & 0.15 \\
\hline Number of females, $n$ & 7 & 10 & 5 & 16 & 0.75 \\
\hline $\mathrm{BMI}$, mean (SD) & $27.8(3.3)$ & $25.1(3.2)$ & $27.0(5.9)$ & $25.6(3.6)$ & 0.37 \\
\hline Charlson comorbidity index, mean (SD) & $2.1(0.4)$ & $3.1(1.8)$ & $3.9(2.2)$ & $3.8(2.0)$ & $<0.001$ \\
\hline Diagnosis, $n$ & & & & & 0.002 \\
\hline Advanced breast cancer & 0 & 3 & 0 & 12 & \\
\hline Soft tissue sarcoma & 5 & 3 & 5 & 3 & \\
\hline Chondro or bone sarcoma & 2 & 5 & 0 & 7 & \\
\hline Others & 1 & 4 & 3 & 2 & \\
\hline Chest wall full-thickness resection, $n$ & 0 & 3 & 2 & 24 & $<0.001$ \\
\hline Mean defect size, $\mathrm{cm}^{2},(\mathrm{SD})$ & $162(63)$ & $130(127)$ & $209(116)$ & $201(128)$ & 0.26 \\
\hline Adjuvant therapy, $n$ & 3 & 5 & 2 & 13 & 0.44 \\
\hline Recurrence during follow-up, $n$ & 0 & 2 & 0 & 2 & 0.71 \\
\hline Metastases during follow-up, $n$ & 0 & 1 & 0 & 3 & 0.81 \\
\hline $\begin{array}{l}\text { Median time after primary surgery, } \\
\text { months, median (range) }\end{array}$ & $\begin{array}{l}92 \\
(16-241)\end{array}$ & $59(18-229)$ & $59(33-207)$ & $73(29-240)$ & 0.44 \\
\hline
\end{tabular}

Table 3 The mean 15D scores and the mean EORTC QLQ-30 function scores of patients who underwent chest wall reconstruction according to reconstruction type group.

\begin{tabular}{|c|c|c|c|c|c|c|}
\hline & \multicolumn{4}{|c|}{ Reconstruction type } & \multicolumn{2}{|c|}{$p$-value } \\
\hline & $\begin{array}{l}\text { Primary } \\
\text { closure } \\
n=8 \\
\text { Mean (SD) }\end{array}$ & $\begin{array}{l}\text { Chest wall } \\
\text { stabilization } \\
n=15 \\
\text { Mean (SD) }\end{array}$ & $\begin{array}{l}\text { Soft tissue } \\
\text { flap cover } \\
n=8 \\
\text { Mean (SD) }\end{array}$ & $\begin{array}{l}\text { Chest wall stabi- } \\
\text { lization + flap } \\
n=24 \\
\text { Mean (SD) }\end{array}$ & Crude & Adjusted \\
\hline \multicolumn{7}{|l|}{ EORTC QLQ-C30: } \\
\hline $\begin{array}{l}\text { Global health status/QoL } \\
\text { Functional scales }\end{array}$ & $79(17)$ & $69(22)$ & $64(27)$ & $76(17)$ & 0.38 & 0.53 \\
\hline Physical & 79 (27) & $74(31)$ & $63(31)$ & $86(14)$ & 0.14 & 0.17 \\
\hline Role & $83(24)$ & $79(29)$ & $65(46)$ & $90(15)$ & 0.24 & 0.35 \\
\hline Emotional & $94(10)$ & $84(18)$ & $74(25)$ & $91(11)$ & 0.095 & 0.29 \\
\hline Cognitive & 92 (18) & $86(22)$ & $81(24)$ & $94(10)$ & 0.25 & 0.58 \\
\hline Social & $98(6)$ & $90(16)$ & $75(33)$ & $95(13)$ & 0.11 & 0.55 \\
\hline \multicolumn{7}{|l|}{ 15D: } \\
\hline Total score & $0.881(0.069)$ & $0.862(0.116)$ & $0.798(0.199)$ & $0.910(0.060)$ & 0.18 & 0.28 \\
\hline Move & $0.907(0.264)$ & $0.943(0.119)$ & $0.871(0.269)$ & $0.921(0.179)$ & 0.89 & 0.93 \\
\hline See & $1.000(0.000)$ & $0.957(0.089)$ & $0.792(0.211)$ & $1.000(0.000)$ & 0.003 & 0.51 \\
\hline Hear & $0.937(0.116)$ & $0.810(0.301)$ & $0.778(0.346)$ & $0.832(0.221)$ & 0.20 & 0.25 \\
\hline Breath & $0.701(0.307)$ & $0.769(0.185)$ & $0.766(0.285)$ & $0.836(0.154)$ & 0.44 & 0.45 \\
\hline Sleep & $1.000(0.000)$ & $0.984(0.062)$ & $1.000(0.000)$ & $1.000(0.000)$ & 0.23 & 0.26 \\
\hline Eat & $1.000(0.000)$ & $0.923(0.220)$ & $0.912(0.164)$ & $1.000(0.000)$ & 0.12 & 0.16 \\
\hline Speech & $0.815(0.154)$ & $0.960(0.104)$ & $0.724(0.278)$ & $0.938(0.123)$ & 0.014 & 0.34 \\
\hline Excretion & $0.921(0.146)$ & $0.777(0.307)$ & $0.770(0.269)$ & $0.897(0.195)$ & 0.28 & 0.19 \\
\hline Usual activities & $0.822(0.215)$ & $0.792(0.315)$ & $0.686(0.308)$ & $0.848(0.167)$ & 0.52 & 0.46 \\
\hline Mental function & $0.911(0.165)$ & $0.929(0.148)$ & $0.822(0.191)$ & $0.926(0.148)$ & 0.53 & 0.64 \\
\hline Discomfort and symptoms & $0.776(0.213)$ & $0.761(0.168)$ & $0.689(0.365)$ & $0.788(0.187)$ & 0.88 & 0.17 \\
\hline Depression & $0.883(0.126)$ & $0.858(0.151)$ & $0.794(0.264)$ & $0.951(0.097)$ & 0.053 & 0.43 \\
\hline Distress & $0.931(0.127)$ & $0.874(0.171)$ & 0.767 (0.219) & $0.966(0.093)$ & 0.024 & 0.84 \\
\hline Vitality & $0.854(0.178)$ & $0.796(0.179)$ & $0.735(0.320)$ & $0.874(0.156)$ & 0.40 & 0.45 \\
\hline Sex & $0.819(0.307)$ & $0.758(0.346)$ & $0.858(0.213)$ & $0.891(0.144)$ & 0.49 & 0.40 \\
\hline
\end{tabular}

a Adjusted for sex, age, and Charlson comorbidity index. 
The extent of the defect correlated only marginally with the generic HRQoL $(r=-0.28 ; 95 \% \mathrm{Cl}:-0.51$ to -0.01 ; $p=0.047$, adjusted for age, gender, and Charlson comorbidity index), but QLQ-C30 correlated strongly with the extent of the defect $(r=-0.37 ; 95 \% \mathrm{Cl}:-0.58$ to $-0.12 ; p=0.007$ adjusted for age, gender, and Charlson comorbidity index).

\section{Discussion}

The main finding of the present study is that long-term HRQoL in patients after chest wall reconstruction following oncological resection is largely comparable to that of the age- and gender-standardized general population. Patients were statistically significantly worse off on the dimensions of "Breathing" and "Usual activities." In different reconstruction type groups, there were no statistically significant differences in HRQoL after analyses were adjusted for sex, age, and Charlson comorbidity index.

There has been limited data on quality of life after chest wall resection and reconstruction compared to those of the healthy control population. In line with our findings, Liu et al. ${ }^{10}$ reported that chest wall resection with pulmonary resection does not worsen HRQoL compared to pulmonary resection without chest wall resection. Their sample size $(n=63)$ was large, but they did not compare results to a sample of age- and gender-standardized general population, and they did not report the reconstruction methods, which can be considered a limitation when reporting the outcomes.

Daigeler et al. ${ }^{9}$ reported that after chest wall reconstruction, quality of life was significantly reduced compared to that in the healthy control group. Their sample size was 36 patients, and they did not analyze the diagnosis of patients, size of the resection, or the reconstruction method in this study group.

Heuker et al. ${ }^{8}$ noticed that subjective assessment of dyspnea was correlated well with patient-perceived HRQoL. Results of our study also indicate that limitation in breathing can occur and HRQoL can be decreased. Heuker et al. did not report in their study $(n=23)$ whether patients had undergone oncological adjuvant treatment, which can have an impact on HRQoL. Tacconi et al. ${ }^{12}$ discovered that the extent of chest wall resection, preoperative forced expiratory volume, and postoperative decline in forced vital capacity were the main indicators for decline in HRQoL as measured by the SF-36. Decline of HRQoL was correlated with the extent of surgical trauma. Likewise, we also noticed in our data the correlation between QLQ-C30 and mean defect size and had similar findings that some limitations in breathing can occur.

A meta-analysis of HRQOL results in patients who underwent chest wall resection treated for recurrent breast cancer was not feasible due to the above-mentioned reporting inconsistencies. ${ }^{13}$ Validated quantitative metrics were used in only one study to report HRQoL. In that study, the authors used The Union for International Cancer Control's (UICC) performance status, but there were only six patients. In that study, the HRQoL improved due to the treatment. ${ }^{11}$ Furthermore, in a study by Toija et al., after treatment of breast cancer (without chest wall resection and reconstruction), the mean 15D HRQoL score was $0.886 .^{24}$ Rautalin et al. found that the mean 15D HRQoL score after treatment of breast cancer is $0.91 .^{25}$ The present study found relatively similar results of mean 15D score of 0.88 among patients who underwent chest wall resection and reconstruction due to malignancy.

The follow-up was long in our study. Median time after primary surgery was 66 months, and the number of respondents in this study was acceptable to draw a conclusion. The sample size is comparable favorably with that of previous studies. ${ }^{9-13}$ The response rate $(71 \%)$ can also be considered acceptable. In our study, we report the extent of surgical operation, need for reconstruction, method of reconstruction, and patient's diagnosis. We have compared our results to those of a large control population.

We acknowledge that there are also some limitations in our study. The most significant limitation is that the study design was not prospective. The heterogeneity of the patients, heterogeneity of the treatments (surgical and oncological), and variability of timing of surgery to completion of questionnaire are also significant limitations in our study, and these could be confounding features. The lack of preoperative assessment negates our ability to assess the real impact of treatment on HRQoL. Comparing results in relatively small reconstruction type subgroups to those of general control population is not reasonable. Possible differences in the subgroups of full- and partial-thickness resections could not be reliably assessed owing to the small sample size. Some of the patients were treated with oncological adjuvant treatment; however, the possible effect could not be analyzed owing to the small sample size. As the study population was heterogeneous, the results could be generalized in patients having undergone chest wall resection and reconstruction for various pathologies. Moreover, comparison of the QLQC30 scores with those of general population could potentially have provided even deeper insight into the impact of chest wall tumor resection and reconstruction to HRQoL. However, this study limited the comparison to the generic HRQoL assessed using the 15D instrument. By the start of the HRQoL data collection in February 2016, 55 (41\%) of the 135 surgical patients who had been operated between 1997 and 2015 had died. These patients could have had most advanced disease, and this fact could have affected the results reported in the present study.

\section{Conclusions}

In conclusion, the main finding of this study is that the longterm HRQoL in patients after chest wall reconstruction following oncological resection is fair and comparable to that of the age- and gender-standardized general population. Limitations in breathing and usual activities can occur. Further prospective studies are needed to assess the true impact of oncological resection and reconstructive surgery in these rare patients.

\section{Conflict of interest}

Each author declares no financial conflicts of interest with regard to the data presented in this manuscript. The funding 
of this article was received from departmental sources only. Dr Harri Sintonen is the developer of the 15D $\odot$.

\section{Acknowledgment}

The authors are thankful for Hannu Kautiainen, MSc, for statistical analysis.

\section{References}

1. Losken A, Thourani VH, Carlson GW, Jones GE, Culbertson JH, Miller JI, et al. A reconstructive algorithm for plastic surgery following extensive chest wall resection. $\mathrm{Br} J$ Plast Surg 2004;57(4):295-302.

2. Mansour KA, Thourani VH, Losken A, Reeves JG, Miller JI, Carlson GW, et al. Chest wall resections and reconstruction: a 25-year experience. Ann Thorac Surg 2002;73(6):6.

3. Tukiainen $\mathrm{E}$. Chest wall reconstruction after oncological resections. Scand J Surg 2013;102(1):9-13.

4. Deschamps C, Tirnaksiz BM, Darbandi R, Trastek VF, Allen MS, Miller DL, et al. Early and long-term results of prosthetic chest wall reconstruction. J Thorac Cardiovasc Surg 1999;117(3):2.

5. Salo JTK, Tukiainen EJ. Oncologic resection and reconstruction of the chest wall: a 19-year experience in a single center. Plast Reconstr Surg 2018;142(2):536-47.

6. Elia S, Griffo S, Gentile M, Costabile R, Ferrante G. Surgical treatment of lung cancer invading chest wall: a retrospective analysis of 110 patients. Eur J Cardiothorac Surg 2001;20(2):356-60.

7. Bottomley A, Aaronson NK. European Organisation for Research and Treatment of Cancer. International perspective on health-related quality-of-life research in cancer clinical trials: the European Organisation for Research and Treatment of Cancer experience. J Clin Oncol. 2007;25(32):5082-6.

8. Heuker D, Lengele B, Delecluse V, Weynand B, Liistro G, Balduyck $B$, et al. Subjective and objective assessment of quality of life after chest wall resection. Eur J Cardiothorac Surg 2011;39(1):102-8.

9. Daigeler A, Druecke D, Hakimi M, Duchna HW, Goertz O, Homann $\mathrm{HH}$, et al. Reconstruction of the thoracic walllong-term follow-up including pulmonary function tests. Langenbecks Arch Surg 2009;394(4):705-15.

10. Liu M, Wampfler JA, Dai J, Gupta R, Xue Z, Stoddard SM, et al. Chest wall resection for non-small cell lung cancer: a case-matched study of postoperative pulmonary function and quality of life. Lung Cancer 2017;106:37-41.

11. Nakao K, Miyata M, Ito T, Ogino N, Kawashima Y, Maeda M, et al. Omental transposition and skin graft in patients for advanced or recurrent breast cancer. Jpn J Surg 1986;16(2):112-17.
12. Tacconi F, Ambrogi V, Mineo D, Mineo TC. The impact on quality of life after en-bloc resection for non-small-cell lung cancer involving the chest wall. Thorac Cancer 2012;3(4):326-33.

13. Wakeam E, Acuna SA, Keshavjee S. Chest wall resection for recurrent breast cancer in the modern era: a systematic review and meta-analysis. Ann Surg 2017.

14. Dindo D, Demartines N, Clavien PA. Classification of surgical complications: a new proposal with evaluation in a cohort of 6336 patients and results of a survey. Ann Surg 2004;240(2):205-13.

15. Koskinen S, Lundqvist A, Ristiluoma N, editors. Health, functional capacity and welfare in Finland in 2011. \&nbsp, Helsinki: National Institute for Health and Welfare (THL); 2012. Report 68/2012.

16. Sintonen $\mathrm{H}$. The 15D instrument of health-related quality of life: properties and applications. Ann Med 2001;33(5):328-36.

17. Stavem K. Reliability, validity and responsiveness of two multiattribute utility measures in patients with chronic obstructive pulmonary disease. Qual Life Res 1999;8(1-2):45-54.

18. Alanne S, Roine RP, Rasanen P, Vainiola T, Sintonen H. Estimating the minimum important change in the $15 \mathrm{D}$ scores. Qual Life Res 2015;24(3):599-606.

19. Hawthorne G, Richardson J, Day NA. A comparison of the Assessment of Quality of Life (AQOL) with four other generic utility instruments. Ann Med 2001;33(5):358-70.

20. Moock J, Kohlmann T. Comparing preference-based quality-of-life measures: results from rehabilitation patients with musculoskeletal, cardiovascular, or psychosomatic disorders. Qual Life Res 2008;17(3):485-95.

21. Richardson J, lezzi A, Khan MA, Chen G, Maxwell A. Measuring the sensitivity and construct validity of 6 utility instruments in 7 disease areas. Med Decis Making 2016;36(2):147-59.

22. Aaronson NK, Ahmedzai S, Bergman B, Bullinger M, Cull A, Duez NJ, et al. The European Organization for Research and Treatment of Cancer QLQ-C30: a quality-of-life instrument for use in international clinical trials in oncology. J Natl Cancer Inst 1993;85(5):365-76.

23. Von Elm E, Altman DG, Egger $M$, et al. The Strengthening the Reporting of Observational Studies in Epidemiology (STROBE) statement: guidelines for reporting observational studies. Lancet 2007;20(370):1453-7.

24. Toija AS, Kettunen TH, Leidenius MHK, Vainiola THK, Roine RPA. Effectiveness of peer support on health-related quality of life in recently diagnosed breast cancer patients: a randomized controlled trial. Support Care Cancer 2019;27(1):123-30.

25. Rautalin M, Färkkilä N, Sintonen H, Saarto T, Taari K, Jahkola T, Roine RP. Health-related quality of life in different states of breast cancer - comparing different instruments. Acta Oncol 2018;57(5):622-8. 